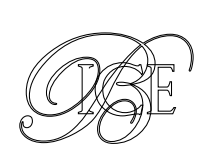

\title{
FINANCIACIÓN DE LOS SERVICIOS PÚBLICOS VINCULADOS A INFRAESTRUCTURAS
}

Las autoridades públicas deberían tener en cuenta las externalidades que generan los servicios públicos vinculados a infraestructuras a la hora de diseñar las políticas encaminadas a maximizar el bienestar, actual y futuro, de la sociedad. En este artículo revisaremos el modelo de financiación de los servicios vinculados a infraestructuras de transporte, primero, y de agua, después, para ver en qué medida dichos modelos generan los incentivos correctos para la minimización de las externalidades negativas asociadas a los mismos. Concluiremos que el sistema de transportes está muy sesgado hacia la carretera, por lo que sería conveniente cobrar a los usuarios de la misma los costes directos y externos que generan a la infraestructura y a la sociedad, respectivamente. De la misma manera, concluiremos que es necesario que las tarifas por los servicios de agua, especialmente por los de depuración de aguas residuales, aseguren su sostenibilidad. Estas políticas deberían venir acompañadas de medidas de protección social para no perjudicar a los colectivos más vulnerables económicamente.

Palabras clave: infraestructuras, transporte, carretera, ferrocarril, Comisión Europea, Pacto Verde Europeo, externalidades, precios, tarifas, tasas, cánones, eficiencia.

Clasificación JEL: H40, H230, Q25, R48.

\section{Introducción}

Bajo los supuestos del modelo de competencia perfecta, los precios de mercado dan las señales correctas para garantizar el equilibrio

\footnotetext{
* Técnico Comercial y Economista del Estado. Vocal asesor en la Dirección General de Política Económica.

El autor agradece a Nieves García Vidal sus aportaciones en materia de agua, así como a Marta Bardón Fernández-Pacheco y Luis Martí Álvarez otras aportaciones en los últimos meses de las que también se enriquece este artículo. No obstante, las opiniones expresadas en este documento son de exclusiva responsabilidad del autor.

Versión de febrero de 2020.

DOI: https://doi.org/10.32796/bice.2020.3121.6980
}

entre la oferta y la demanda de los distintos bienes y servicios. Además, los precios de equilibrio del mercado proporcionan tanto a los vendedores como a los compradores relaciones de intercambio en el mercado que pueden utilizar como parámetros para tomar sus decisiones. Al ser estas relaciones de intercambio las mismas para todos, queda garantizada la eficiente asignación de los recursos. Las fuerzas de la oferta y la demanda conducen a la combinación eficiente. Por ello, la intervención pública en el mercado no puede más que provocar una pérdida de eficiencia en la asignación de los recursos (Nicholson, 1997). 
Sin embargo, el mundo académico también ha teorizado sobre diversas situaciones en las que no se cumplen los supuestos en los que se apoya el modelo. Una de las más estudiadas es la de las externalidades. Estas se producen cuando un agente lleva a cabo una acción de la cual se derivan unos beneficios o costes para otros agentes. La característica fundamental de las externalidades es que el agente causante de los efectos externos no está obligado a realizar ningún pago en concepto de indemnización a los afectados a quienes ha impuesto costes, o no tiene derecho a recibir una compensación por los beneficios generados (De Rus et al., 2003). En presencia de externalidades, las decisiones de los agentes no darán lugar a una asignación óptima de los recursos (Nicholson, 1997). Si los productores de determinados bienes y servicios no internalizan los beneficios o costes que provocan a terceros, los supuestos del modelo de competencia perfecta no se cumplen y, necesariamente, el mercado generará un nivel de producción por debajo o por encima del socialmente óptimo, respectivamente.

Algunos servicios públicos generan unas externalidades positivas. Por ejemplo, el acceso de un ciudadano a la asistencia médica y, en su caso, el consumo de fármacos bajo control médico contribuyen a evitar la propagación de enfermedades infecciosas al resto de la población. Esto justifica -no solo por motivos de equidad, sino incluso por motivos de eficiencia- la intervención pública en el ámbito de la salud, subvencionando la provisión de servicios médicos y hospitalarios y el consumo controlado de fármacos, para evitar un uso de los mismos que sea inferior al socialmente óptimo que se derivaría de una asignación de recursos puramente de mercado.

En el caso de los servicios públicos vinculados a infraestructuras, también se producen externalidades positivas. Por ejemplo, las de saneamiento y depuración de aguas residuales también contribuyen a evitar las enfermedades infecciosas. No obstante, las propias infraestructuras suelen tener un impacto medioambiental negativo importante. Por ejemplo, los efectos barrera para la fauna, incluso por parte de líneas ferroviarias o carreteras en desuso, el movimiento de tierras para la construcción, residuos de materiales de construcción, la modificación de costas y cauces fluviales (De Rus et al., 2003). Por su parte, los servicios que se prestan sobre o con ellas también presentan externalidades negativas —como la contaminación atmosférica, la emisión de gases de efecto invernadero, vehículos obsoletos descartados, los aceites usados, el ruido, la siniestralidad, la posibilidad de vertidos de petróleo o de sustancias contaminantes, la congestión (De Rus et al., 2003), o el agotamiento de un recurso escaso y valioso como es el agua-. Todas estas externalidades deben ser tenidas muy en cuenta por las autoridades públicas a la hora de diseñar las políticas encaminadas a maximizar el bienestar, actual y futuro, de la sociedad.

\section{Pago por el uso de infraestructuras de transporte}

\subsection{Situación actual}

La mayor parte de las carreteras se ha financiado con cargo al gasto público, aunque con las primeras autopistas que se construyeron en los años sesenta-setenta se utilizó el modelo de concesión de autopista de peaje, que se volvió a utilizar a principios de los años 2000. En 2017, en España existían 165.686 km de carreteras. De estas, un total de $17.163 \mathrm{~km}$ se podían considerar vías de alta capacidad $\triangleright$ 
(Observatorio del Transporte y la Logística en España, 2019) ${ }^{1}$. Tras la supresión de los peajes en la AP-1 entre Burgos y Armiñón, en la AP-4 entre Sevilla y Cádiz, y en la AP-7 entre Tarragona y Alicante, quedan un total de $2.755,1 \mathrm{~km}$ en régimen de autopistas de peaje (Delegación del Gobierno en las Sociedades Concesionarias de Autopistas Nacionales de Peaje, 2018), lo que supone un $1,66 \%$ de las carreteras totales y un $16,1 \%$ de las vías de alta capacidad. Por lo tanto, podemos afirmar que el grueso de las infraestructuras viarias en España es gratuito para los usuarios y se financia con cargo a los contribuyentes.

Esto contrasta con lo que ocurre con los demás modos de transporte, ya que los operadores ferroviarios tienen que pagar cánones ferroviarios a ADIF y a ADIF Alta Velocidad por el uso de sus infraestructuras, las aerolíneas tienen que pagar tarifas aeroportuarias a AENA para poder utilizar los aeropuertos y las navieras tienen que pagar tasas portuarias a las distintas autoridades portuarias para poder utilizar los puertos.

El Cuadro 1 muestra, por ejemplo, los cánones actualmente en vigor que tienen que pagar los trenes por utilizar las vías férreas.

Aunque las tarifas aeroportuarias no son las mismas para todos los aeropuertos - hay diferentes categorías de aeropuertos a los que se les aplican distintos niveles tarifarios en función de su volumen de tráfico y de su carácter o no de aeropuertos insulares-, el Cuadro 2 muestra las tarifas medias unitarias cobradas en 2018 por AENA, según la Comisión Nacional de los Mercados y la Competencia, por cada prestación patrimonial pública reconocida en la Ley de Seguridad Aérea.

\footnotetext{
1 Las vías de alta capacidad incluyen autopistas de peaje, autopistas libres de peaje y autovías y carreteras multicarril.
}

El Cuadro 3 resume los pagos que se realizan en España por el uso de infraestructuras de transporte.

Aunque los casi 1.500 millones de euros de ingresos por peajes de las sociedades concesionarias de autopistas podrían parecer muchos en comparación con los cargos cobrados por ADIF, ADIF AV y las autoridades portuarias por el uso de sus respectivas infraestructuras, estos cargos se deberían poner en relación con los volúmenes de tráfico que soportan las mismas.

Estos volúmenes de tráfico no son siempre fáciles de comparar, por ejemplo, porque incluyen tanto tráfico de viajeros como de mercancías y no es fácil averiguar la proporción que representa cada tipo de tráfico sobre el total de ingresos. De la misma manera, por el tipo de infraestructura de la que se trata (los kilómetros que recorre cada tonelada o viajero sobre la infraestructura), los ingresos generados por el tráfico en las carreteras y los ferrocarriles son más comparables entre sí que con los generados por el tráfico en puertos y aeropuertos, que, a su vez, serán más comparables entre sí que con los dos primeros tipos de infraestructuras mencionadas. A pesar de estas importantes limitaciones, podemos tratar de elaborar algunas ratios para tener algún indicio de en qué medida el pago por el uso de las infraestructuras viarias puede estar al mismo nivel o por debajo del que se produce en el resto de las infraestructuras de transporte. En el Cuadro 4 observamos el número de toneladas de mercancías (tanto en el tráfico interior como en el internacional) que pasan por las infraestructuras españolas y las ponemos en relación con los ingresos expuestos en el Cuadro 3.

Se observa que los ingresos de los operadores de infraestructuras de carreteras por tonelada transportada son los menores de $D$ 
CUADRO 1

CÁNONES FERROVIARIOS VIGENTES

(En euros/tren-km o euros/100 plazas-km, según los casos)

\begin{tabular}{|c|c|c|c|c|c|c|}
\hline Cánones y recargos cobrados por ADIF Alta Velocidad & VL1 & VL2 & VL3 & VCM & VOT & M \\
\hline Canon por adjudicación de capacidad (euros tren-km) & & & & & 0,485 & ,4446 \\
\hline $\begin{array}{l}\text { Adición al canon por adjudicación de capacidad por el uso no } \\
\text { eficiente de ésta (euros tren-km) }\end{array}$ & 11,0201 & 3,9888 & 8,4803 & 4,421 & 1,985 & 1,7356 \\
\hline Canon por utilización de las líneas ferroviarias (euros tren-km) & & & & & 1,25 & 1,1055 \\
\hline $\begin{array}{l}\text { Adición al canon por utilización de las líneas ferroviarias por el uso } \\
\text { de redes de altas prestaciones o la explotación de servicios de ancho } \\
\text { variable u otras situaciones de elevada intensidad de tráfico en } \\
\text { determinados periodos horarios en la línea Madrid-Barcelona- } \\
\text { frontera francesa (euros por cada } 100 \text { plazas-km ofertadas) }\end{array}$ & 1,7611 & 0,2317 & 0,3023 & 0,496 & 0 & 0 \\
\hline $\begin{array}{l}\text { Adición al canon por utilización de las líneas ferroviarias por el uso } \\
\text { de redes de altas prestaciones o la explotación de servicios de ancho } \\
\text { variable u otras situaciones de elevada intensidad de tráfico en } \\
\text { determinados periodos horarios en la línea Madrid-Toledo-Sevilla- } \\
\text { Málaga (euros por cada } 100 \text { plazas-km ofertadas) }\end{array}$ & 0,8647 & 0,1504 & 0,1962 & 0,322 & 0 & 0 \\
\hline $\begin{array}{l}\text { Canon por utilización de las instalaciones de transformación y } \\
\text { distribución de la energía eléctrica de tracción (euros tren-km) }\end{array}$ & 0,802 & 0,3835 & 0,802 & 0,395 & 0,25 & 0,1855 \\
\hline Cánones y recargos cobrados por ADIF en euros tren-km & VL1 & VL2 & VL3 & & & M \\
\hline Canon por adjudicación de capacidad & 0,5082 & & & & & \\
\hline $\begin{array}{l}\text { Adición al canon por adjudicación de capacidad por el uso no } \\
\text { eficiente de ésta }\end{array}$ & 1,4346 & 1,4492 & 1,445 & 6,27 & 1,161 & 0,2043 \\
\hline Canon por utilización de las líneas ferroviar & 0,7247 & 0,732 & 0,7299 & 1,975 & 0,5865 & 0,1032 \\
\hline $\begin{array}{l}\text { Adición al canon por utilización de las líneas ferroviarias por el uso } \\
\text { de redes de altas prestaciones o la explotación de servicios de ancho } \\
\text { variable u otras situaciones de elevada intensidad de tráfico en } \\
\text { determinados periodos horarios }\end{array}$ & 0 & 0 & 0 & 2,36 & 0 & 0 \\
\hline $\begin{array}{l}\text { Canon por utilización de las instalacione } \\
\text { distribución de la energía eléctrica de tr }\end{array}$ & 0,2018 & 0,2039 & 0,2033 & 0,55 & 0,1635 & 0,0287 \\
\hline \multicolumn{7}{|l|}{ Tipos de servicio: } \\
\hline \multicolumn{7}{|c|}{$\begin{array}{l}\text { VL1: servicios de larga distancia, excepto los designados como VL2, VL3 y VOT. } \\
\text { VL2: servicios de larga distancia en relaciones de ancho variable, siempre que al menos un } 10 \% \text { de su recorrido total discurra por líneas de ancho ibérico, } \\
\text { excluidos los designados como VL3. } \\
\text { VL3: servicios de larga distancia en relaciones transversales largas: recorridos superiores a } 700 \text { kilómetros y que no tengan origen, destino o parada } \\
\text { intermedia en Madrid y sus ramas. } \\
\text { VCM: servicios de viajeros urbanos o suburbanos e interurbanos. } \\
\text { VOT: trenes y material de viajeros sin pasajeros, incluidas máquinas aisladas, movimiento de trenes en vacío, formación y pruebas. } \\
\text { M: servicios de mercancías: todos los servicios de mercancías, incluidos los cargados, los vacíos y pruebas. }\end{array}$} \\
\hline \multicolumn{7}{|l|}{ Fuente: Ley 6/2018, de 3 de julio, de Presupuestos Generales del Estado para el año 201} \\
\hline
\end{tabular}

todos los modos de transporte. Además, el año de comparación, 2017, es previo a un incremento notable en 2018 de los cánones recaudados por ADIF, sin que se hayan producido variaciones significativas en el tráfico, por lo que los 32,76 euros recaudados por tonelada, probablemente, infraestiman los niveles actuales de cobro por el uso de las infraestructuras ferroviarias. No obstante, en el caso de los ferrocarriles y los aeropuertos, la comparación estará muy probablemente sesgada por la elevada proporción que representan los viajeros en los ingresos totales de ADIF, ADIF Alta Velocidad y AENA. Por ello, también podemos comparar la recaudación total por uso de infraestructuras viarias y ferroviarias con el número de viajeros-km en viajes interiores en España.

Vemos que, tomando como referencia el transporte de viajeros, el pago unitario por uso de infraestructuras, aunque en menor medida que en el caso de las mercancías, sigue $\square$ 
CUADRO 2

PERCEPCIONES MEDIAS POR PRESTACIONES PATRIMONIALES PÚBLICAS DE AENA EN 2018

(En euros)

\begin{tabular}{|l|c|c|}
\hline \multicolumn{1}{|c|}{ Prestación patrimonial pública } & Euros & Unidad \\
\hline Pasajeros & 4,36 & pasajero \\
Seguridad & 1,61 & pasajero \\
Servicios de PMR & 0,29 & pasajero \\
Catering & 0,04 & pasajero \\
Aterrizaje & 211,11 & operación de aeronave \\
Servicios meteorológicos & 5,59 & operación \\
Servicio de tránsito de aeródromo & 101,02 & operación \\
Utilización de pasarelas telescópicas & 46,44 & operación \\
Handling & 43,66 & operación \\
Combustible de aviación & 14,62 & operación \\
Estacionamiento & 16,26 & operación \\
\hline Fuente: Comisión Nacional de los Mercados y de la Competencia, 2019. & & \\
\hline
\end{tabular}

CUADRO 3

COBROS A USUARIOS POR LA UTILIZACIÓN DE INFRAESTRUCTURAS DE TRANSPORTE

\begin{tabular}{|l|r|r|}
\hline \multicolumn{1}{|c|}{ Gestor de infraestructuras } & \multicolumn{2}{c|}{ Ingresos por año (miles de euros) } \\
\cline { 2 - 3 } & $\mathbf{2 0 1 7}$ & $\mathbf{2 0 1 8}$ \\
\hline AENA (prestaciones patrimoniales públicas en la red de aeropuertos) & 2.562 .051 & 2.676 .491 \\
ADIF (ingresos por liquidación de cánones ferroviarios) & 378.997 & 659.014 \\
ADIF Alta Velocidad (ingresos por liquidación de cánones ferroviarios) & 548.794 & 572.092 \\
Sistema portuario (tasas portuarias) & 1.017 .900 & 1.041 .487 \\
Sociedades concesionarias de autopistas de peaje (ingresos por peajes) & 1.487 .439 & n.d. \\
\hline $\begin{array}{l}\text { Fuente: AENA (2019), ADIF (2019), ADIF Alta Velocidad (2019), Puertos del Estado (2019) y Delegación del Gobierno en las Sociedades } \\
\text { Concesionarias de Autopistas Nacionales de Peaje (2018). }\end{array}$ \\
\hline
\end{tabular}

CUADRO 4

INGRESOS POR INFRAESTRUCTURAS DE TRANSPORTE COMPARADOS CON EL TRANSPORTE DE MERCANCÍAS

\begin{tabular}{|l|c|c|}
\hline \multicolumn{1}{|c|}{ Modo } & Miles de toneladas en $\mathbf{2 0 1 7}$ & Ingresos en euros por infraestructuras/toneladas \\
\hline Carretera & 1.441 .365 & 1,031965 \\
Ferroviario & 28.317 & 32,764452 \\
Aéreo & 865 & $2.961,908671$ \\
Marítimo & 493.413 & 2,062977 \\
\hline $\begin{array}{l}\text { Fuente: Observatorio del Transporte y la Logística en España, 2019, y elaboración propia a partir de la fuente anterior y de AENA, ADIF, ADIF Alta } \\
\text { Velocidad y Delegación del Gobierno en las Sociedades Concesionarias de Autopistas Nacionales de Peaje. }\end{array}$
\end{tabular}

CUADRO 5

INGRESOS POR INFRAESTRUCTURAS DE TRANSPORTE COMPARADOS CON EL TRANSPORTE DE VIAJEROS

\begin{tabular}{|l|c|c|}
\hline \multicolumn{1}{|c|}{ Modo } & Millones de viajeros-km en $\mathbf{2 0 1 7}$ & Ingresos por infraestructura/viajero.km (cts de euro) \\
\hline Carretera & 368.717 & 0,4034094 \\
\hline Ferroviario & 27.387 & 3,3877058 \\
\hline \multicolumn{2}{|l|}{ Fuente: Observatorio del Transporte y la Logística en España, 2019, y elaboración propia a partir de la fuente anterior y de AENA, ADIF, ADIF Alta } \\
Velocidad y Delegación del Gobierno en las Sociedades Concesionarias de Autopistas Nacionales de Peaje.
\end{tabular}


siendo muy superior en el caso de los ferrocarriles que en el caso de las carreteras ${ }^{2}$. Estos datos omiten los recorridos internacionales de viajeros, pero esta omisión no es importante porque la relación entre viajeros-km en el transporte por carretera, en comparación con el transporte por ferrocarril, está claramente más descompensada hacia la carretera en los viajes internacionales que en los interiores -lo cual es, por otra parte, lógico teniendo en cuenta el condicionante de los anchos de vías que se utilizan en España-.

Por lo tanto, aunque todas las estimaciones que hemos realizado son imperfectas, siempre apuntan hacia un menor cobro a los usuarios de las carreteras que a los de las infraestructuras de los demás modos de transporte. Además, como hemos visto, el pago de peajes se concentra en unas carreteras que representan una parte minoritaria de la red viaria, lo que supone una mayor dispersión en los precios pagados por los usuarios de las distintas vías.

\subsection{Las externalidades en los distintos modos de transporte}

Esta mayor asequibilidad del transporte viario contrasta con las consecuencias de este modo en comparación con otros. El uso de las carreteras está asociado a unas externalidades negativas económicas y medioambientales muy superiores a las de otros medios de transporte -en particular en comparación con el ferrocarril- por contaminación atmosférica, emisión de gases de efecto invernadero, congestión y siniestralidad.

2 Y además, como ya se ha indicado, en 2018 habrán aumentado todavía más.
El sector del transporte representa el $42,4 \%$ del consumo de energía final en España en 2016, 11,2 puntos porcentuales por encima de la media de los países de la Unión Europea, sin duda por el sesgo de nuestro sistema de transporte hacia la carretera. El transporte de viajeros por ferrocarril es, en términos de consumo energético por unidad-km transportada, tres veces más eficiente que por carretera y el transporte de mercancías por ferrocarril es cinco veces más eficiente (Observatorio del Transporte y la Logística en España, 2019). Esto permite hacerse una idea de cuán distinto es el impacto de estos modos de transporte en la emisión de partículas contaminantes y en la de gases de efecto invernadero, y cómo una asignación eficiente de los recursos parecería deber primar el empleo del modo ferroviario sobre el viario ${ }^{3}$.

De la misma manera, en lo que se refiere a la siniestralidad, en España el número total de accidentes con víctimas fue de 102.233 en las carreteras españolas en 2017, mientras que fue de 167 en el caso del modo ferroviario. Así mismo, el número de víctimas mortales (1.830) también fue mucho mayor en el modo viario que en el ferroviario (29), según el Observatorio del Transporte y la Logística (2019). Por supuesto, estas cifras deben ser puestas en relación con el mayor tráfico que se produce en $\triangleright$

3 Aunque el informe anual del Observatorio también realiza una estimación de las emisiones unitarias de gases de efecto invernadero de cada modo, no lo recojo en este artículo porque podría existir cierto riesgo de sobreestimación de la diferencia entre ambos modos. El Observatorio solo toma en consideración las emisiones producidas por los trenes que consumen diésel y asumen unas emisiones nulas por parte de los trenes eléctricos. Sin embargo, incluso aunque ADIF y ADIF Alta Velocidad, como proveedores de la energía de tracción, exigen a sus comercializadores de energía eléctrica certificados de garantía del origen renovable de la energía que les suministran, habría que ver en qué medida dichas adquisiciones de energía limpia desplazan a fuentes convencionales del mercado y en qué medida simplemente generan un desplazamiento de la asignación contable del consumo de energía procedente de fuentes convencionales a otros consumidores. Probablemente, el desplazamiento de las energías convencionales del mercado no sea total y haya cierto grado de emisiones de gases de efecto invernadero que simplemente se desplazan a otros consumidores que no exigen estos certificados de garantía de origen. 
el modo viario, en comparación con el ferroviario. Sin embargo, aun así se obtiene que el número de víctimas mortales por cada viajero$\mathrm{km}$ sigue siendo casi cinco veces mayor en la carretera que en el modo ferroviario ${ }^{4}$. Si realizamos el ejercicio con el número de accidentes con víctimas, aunque no sean mortales, el resultado comparativo de la carretera que se obtiene es mucho peor, como se puede observar en el Cuadro 6.

Por lo tanto, como vemos, teniendo en cuenta las importantes externalidades negativas que genera el tráfico por carretera, las decisiones de política de transportes en relación al cobro por el uso de las infraestructuras no están contribuyendo a una distribución modal eficiente de la movilidad en España, sino más bien al contrario, suponen un incentivo a una mayor contaminación y siniestralidad.

\subsection{Agenda comunitaria en relación a las externalidades del transporte}

Como consecuencia de estas mayores externalidades por parte del transporte por carretera en comparación con otros modos, el 28 de marzo de 2011, la Comisión adoptó su Libro Blanco, Hoja de ruta hacia un espacio único europeo de transporte: por una política de transportes competitiva y sostenible, que incluye,

4 Aunque existen otros indicadores para comparar la siniestralidad de los modos de transporte (De Rus et al., 2003), tomo los viajeros-km por considerarlo el criterio más objetivo. entre sus diez objetivos para un sistema de transporte competitivo y sostenible, intentar transferir a otros modos, como el ferrocarril o la navegación fluvial, de aquí a 2030, el $30 \%$ del transporte de mercancías por carretera (Comisión Europea, 2011).

Más recientemente, la Comisión Europea hizo público, el 11 de diciembre de 2019, su hoja de ruta en materia de cambio climático, el Pacto Verde Europeo. En él se reitera la prioridad de reconducir una parte sustancial del actual transporte de mercancías por carretera a los modos ferroviario y fluvial. Para ello, la Comisión propondrá en 2021 medidas para incrementar la capacidad de estos dos modos. También se revisará la Directiva de transporte combinado para asegurar que es una herramienta eficaz para apoyar el transporte multimodal utilizando esos dos modos de transporte. Otra de las prioridades declaradas es la de revisar las políticas de precios en las carreteras, por medio de una revisión de la «Directiva Euroviñeta» ${ }^{5}$ con un elevado nivel de ambición (Comisión Europea, 2019c).

Sin embargo, como hemos visto, las decisiones de política de transportes en relación con el cobro por el uso de las infraestructuras no están contribuyendo a una distribución modal eficiente de la movilidad en España, sino más bien al contrario, suponen un incentivo a una mayor contaminación y siniestralidad $\triangleright$

5 Directiva1999/62/CE, relativa a la aplicación de gravámenes a los vehículos pesados de transporte de mercancías por la utilización de determinadas infraestructuras, con sus revisiones posteriores.

CUADRO 6

SINIESTRALIDAD DE LA CARRETERA Y DE LOS FERROCARRILES

\begin{tabular}{|l|c|c|}
\hline \multicolumn{1}{|c|}{ Modo } & $\begin{array}{c}\text { Víctimas mortales por cada } \\
\text { mil millones de viajeros-km en 2017 }\end{array}$ & $\begin{array}{c}\text { Accidentes con víctimas por cada } \\
\text { mil millones de viajeros-km en 2017 }\end{array}$ \\
\hline Carretera & 5,234366737 & 277,2668469 \\
Ferroviario & 1,058896557 & 6,0977836 \\
\hline Fuente: elaboración propia a partir del Observatorio del Transporte y la Logística en España, 2019.
\end{tabular}

Fuente: elaboración propia a partir del Observatorio del Transporte y la Logística en España, 2019. 
por el sesgo a favor de la carretera. Dado que el transporte fluvial en España es inviable, y teniendo en cuenta los condicionantes orográficos e históricos del sector ferroviario (por la existencia de anchos de vía distintos al más habitual a escala internacional), será imposible avanzar hacia los objetivos del Libro Blanco y del Pacto Verde Europeo si no se produce un cambio profundo en las políticas de movilidad de nuestro país. De hecho, en la mayor parte de los países de nuestro entorno ya está mucho más generalizado que en España el pago por el uso de las vías de alta capacidad, especialmente por parte de los vehículos pesados (ATKearney, 2018) ${ }^{6}$. Por lo tanto, avanzar hacia los objetivos comunitarios pasa por lograr una tarificación económicamente más racional, que discrimine según las emisiones que generan los vehículos. Con ello, se promueve la renovación de la flota hacia vehículos menos contaminantes y la traslación del transporte de mercancías por carretera hacia el ferrocarril. Teniendo en cuenta que el desgaste al que someten a las infraestructuras es mucho mayor $y$ que también generan, por vehículo-km, unas emisiones mucho mayores de partículas, para que este cambio de políticas responda efectivamente a las justificaciones planteadas, los vehículos pesados deberían soportar unas tarifas mayores que los ligeros. La preocupación por la competitividad de este sector se podría abordar por medio de un calendario claro y gradual de aproximación a los precios que les corresponda pagar a estos vehículos, de tal manera que el sistema de transportes pueda ir adaptándose a nuevas pautas de comportamiento que sean más sostenibles.

\footnotetext{
6 En países como Alemania, Austria, Bélgica, Bulgaria, Croacia, Dinamarca, Eslovaquia, Eslovenia, Estonia, Hungría, Letonia, Lituania, Noruega, Países Bajos, Polonia, Portugal, Reino Unido, República Checa, Rumanía, Suecia y Suiza, los vehículos pesados pagan por el uso en el $100 \%$ de las vías de alta capacidad (ATKearney, 2018).
}

\subsection{La dimensión presupuestaria}

La generalización del cobro por el uso de las infraestructuras viarias permitiría, en primer lugar, aliviar la carga que supone el coste del mantenimiento de esta red sobre los contribuyentes españoles, que no necesariamente tienen por qué tener recursos económicos suficientes para permitirse un vehículo privado. Por ello, financiar la red viaria con cargo a los usuarios, cuya capacidad contributiva queda revelada por el mero hecho de desplazarse, podría ser más equitativo que hacerlo con cargo a los contribuyentes. La transposición actual de la Directiva Euroviñeta permitiría recuperar estos costes. Esto supondría una contribución, aunque modesta, al alivio de la falta de disponibilidad presupuestaria para abordar otras necesidades crecientes de la sociedad actual como las políticas sociales y la reducción de la deuda pública.

Adicionalmente, si se tomaran las medidas reglamentarias necesarias, se podrían incluso recaudar recursos adicionales para recuperar el coste de las externalidades que generan las carreteras por contaminación atmosférica y ruido (en la medida en que no se están recuperando ya por la vía de los impuestos especiales sobre los hidrocarburos) ${ }^{7}$. Para ello, sería necesario transponer el artículo 7 quater de la Directiva Euroviñeta en la redacción dada por la Directiva 2011/76/UE, un artículo de transposición voluntaria y que España decidió no incorporar en el ordenamiento jurídico español. Sin embargo, la eficiencia económica y medioambiental parecerían justificar que sí se haga en el futuro. Con estos recursos se podrían financiar medidas que permitan mejorar la competitividad del ferrocarril $\triangleright$

7 El hecho de que dichos impuestos son inferiores a los de los países de nuestro entorno supone un indicio de que por lo menos parte de las externalidades generadas no se está cobrando a quienes la generan. 
o acelerar el despliegue en nuestras carreteras del uso de los vehículos eléctricos ${ }^{8}$ y aliviar la carga que supone la siniestralidad sobre el presupuesto del Sistema Nacional de Salud o sobre el de la Dirección General de Tráfico.

Por lo tanto, estamos planteando incrementar la dotación presupuestaria de actuaciones en materia ferroviaria. Sin embargo, la asignación de los recursos que se doten para las actuaciones destinadas a potenciar este modo de transporte no debe quedar exenta de un riguroso análisis de la eficiencia de este modo, en comparación con las alternativas posibles. Una revisión integral de las políticas de movilidad nacionales, autonómicas y locales para asegurar que los trenes aprovechan el máximo de su potencial permitiría, sin duda, la sustitución del transporte privado, y de otros modos más contaminantes de transporte público, por el ferrocarril, en aquellos casos en los que sea posible y eficiente hacerlo. Sin embargo, no en todos los casos el ferrocarril será el modo de transporte más eficiente para responder a las necesidades de movilidad de los ciudadanos. Por ello, no siempre la mejora de las políticas de movilidad españolas pasarán por poner más trenes en circulación, o incluso por mantener los que ya circulan, ya que, por mucho que en teoría los trenes sean más eficientes que los automóviles desde el punto de vista medioambiental, un tren que circula contaminará siempre más que un tren parado. Por ello, en aquellos casos en los que los trenes no sean capaces de atraer a

8 Los vehículos eléctricos tienen la ventaja de que sus motores no generan contaminación atmosférica y no emiten gases de efecto invernadero, por lo menos no directamente. Indirectamente, emiten gases de efecto invernadero en una cantidad que dependerá del peso de las diferentes tecnologías en el sistema eléctrico. Asimismo, mientras siga habiendo centrales nucleares activas, se les debería imputar cierto coste externo por la gestión de residuos nucleares. Por otro lado, la gestión de las baterías usadas de los vehículos eléctricos tendrá un coste medioambiental importante. En este artículo, se presupone que el coste medioambiental de los vehículos eléctricos, en conjunto, es menor que el de los vehículos convencionales. los viajeros y circulen con una ocupación muy por debajo de su capacidad, la política más eficiente no será la de crear circulaciones ferroviarias adicionales o mantener las existentes, sino sustituirlas por servicios utilizando aquellos vehículos más pequeños que sean más eficientes para responder a las necesidades del número de viajeros implicados en cada caso.

\subsection{La mejora de la seguridad}

Las carreteras necesitan recuperarse de los efectos de la falta de financiación de inversión en los últimos años. La ausencia de inversión necesaria en conservación, mantenimiento y gran reparación, así como la falta de estabilidad de las inversiones, se ha traducido en los últimos años en un grave deterioro del patrimonio de las carreteras. La situación de la financiación en materia de carreteras puede calificarse como crítica. Para recuperar el retraso acumulado en los últimos años en materia de conservación, mantenimiento y rehabilitación de carreteras, la inversión debería elevarse al entorno del $0,6 \%$ del PIB en los próximos años, frente al 0,34\% que representó en el año 2016. En caso de seguir retrasándose este esfuerzo, su magnitud puede aumentar exponencialmente (Asociación de Ingenieros de Caminos, Canales y Puertos y de la Ingeniería Civil, 2019). La generalización de un pago por uso a la Administración pública responsable de cada carretera podría contribuir a garantizar su mantenimiento en un estado de conservación óptimo.

\subsection{Las vías urbanas}

En este artículo nos hemos centrado en el cobro por uso en carreteras interurbanas, $\square$ 
pero existen experiencias que han tenido resultados positivos también en accesos a las ciudades, basándose en trabajos académicos del profesor de la Universidad de Columbia William Vickrey a partir de 1952. Los conductores de Singapur están obligados a pagar peajes por entrar en el distrito financiero desde 1975. Este sistema, con sus modificaciones posteriores, ha servido para reducir la congestión, ha incentivado un incremento del uso del transporte público, ha reducido las emisiones de gases de efecto invernadero y ha tenido un impacto recaudatorio significativo para la Administración pública local. Por todo ello, ha sido emulado por ciudades europeas como Londres, Estocolmo y Milán (Rodrik, 2015).

\section{Recuperación de costes en la depuración de aguas residuales urbanas}

\subsection{Marco normativo y competencial}

Como es sabido, la Directiva 2000/60/CE del Parlamento Europeo y del Consejo de 23 de octubre de 2000, por la que se establece un marco comunitario de actuación en el ámbito de la política de aguas (DMA) y otras más específicas que la complementan, está dirigidas a proteger los recursos hídricos existentes en la UE frente a distintos tipos de contaminación o riesgos, para que alcancen un buen estado, así como a promover un uso más racional, eficiente y sostenible del agua, lo que resulta coherente con el objetivo 6 -garantizar la disponibilidad de agua, su gestión sostenible y el saneamiento- de la Agenda 2030 sobre Desarrollo Sostenible, adoptada en 2015 por Naciones Unidas.

Atendiendo a criterios diversos (biológicos y químicos, entre otros), la DMA establece objetivos ambientales para las distintas masas de agua superficiales y subterráneas que los Estados miembros (EE MM) deberán lograr en 2027. También estipula principios y directrices comunes para gestionar el agua, con un enfoque integrado para tener en cuenta las implicaciones ambientales, económicas, sociales y sanitarias.

En España, la normativa de aguas está adaptada a la europea en la materia, en particular a la DMA, mediante el Texto Refundido de la Ley de Aguas (TRLA) aprobado por Real Decreto Legislativo 1/2001 y desarrollado por disposiciones diversas. Ese marco es estatal, lo dicta el Estado como básico (mínimo común), pero su aplicación territorial corresponde a las comunidades autónomas (CC AA) y a las entidades locales, que, además, pueden aprobar normativa adicional.

En principio, los servicios urbanos del agua son municipales, en concreto los del abastecimiento domiciliario de agua potable (suministro en baja), el alcantarillado y, en algunos casos, la depuración, si cuenta con la aprobación de la comunidad autónoma, y son los municipios quienes deciden cómo prestar tales servicios.

Por su parte, las CC AA tienen competencias propias, como las de depuración, y pueden dictar normativa que afecte a los servicios urbanos del agua e incidan, por ejemplo, en su régimen financiero, la calidad del agua para el abastecimiento o los vertidos.

Lo anterior explica la heterogeneidad que caracteriza al sector, en particular las diferencias entre lo que pagan los usuarios urbanos por el agua en función del lugar donde residan y las condiciones que haya establecido cada autoridad implicada, incluidas las de traslación de costes a tarifas. 


\subsection{Problemas para su cumplimiento en España y tarifas para los usuarios}

La Comisión Europea publicó, el 26 de febrero de 2019, su quinto informe sobre la aplicación de la Directiva 2000/60/CE Marco del Agua (DMA) y normativa asociada, que está acompañado de múltiples documentos de trabajo, entre ellos uno sobre España. De entre sus conclusiones, la principal es que, a pesar de los avances logrados y lo previsto en los vigentes planes hidrográficos 2015-2021, queda mucho por hacer de cara a 2027. En la elaboración de los próximos planes 2021-2027, España debería impulsar lo preciso para que todas las masas de agua alcancen el buen estado exigible - pues aún hay varias que no resultan conformes-, lo que exigirá, entre otras cosas, solucionar los incumplimientos de la Directiva 91/271/CEE sobre depuración de aguas residuales urbanas (Comisión Europea, 2019b), en nueve aglomeraciones urbanas con más de 15.000 habitantes equivalentes $^{9}$, por los que España ha sido sancionada (Tribunal de Justicia de la Unión Europea, 2018); o puede serlo por los procedimientos de infracción que tenemos abiertos en la materia (Comisión Europea, 2017). Así mismo, para asegurar la correcta aplicación de la DMA, la recuperación de costes por los servicios del agua sigue siendo baja o mejorable en algunas de nuestras demarcaciones hidrográficas (Comisión Europea, 2019b).

También en este caso, la ausencia de inversión en conservación, mantenimiento y gran reposición ha generado un importante deterioro de las infraestructuras (Asociación de Ingenieros de Caminos, Canales y Puertos y de la Ingeniería Civil, 2019). El 26\% de las redes de

9 Al principio eran 38 aglomeraciones las que no cumplían. abastecimiento y el $44 \%$ de las de alcantarillado superan los cuarenta años y sus porcentajes globales de renovación se sitúan en el 0,6 y en el 0,38 , respectivamente, frente a la tasa recomendada del 2\% (Asociación Española de Empresas Gestoras de los Servicios de Agua Urbana, 2018). Esta falta de mantenimiento impide que el desperdicio de agua por evaporación, fugas y otros motivos se reduzcan al mínimo inevitable. La cobertura de la red de saneamiento no alcanza el $70 \%$ y es inadecuada para cumplir los objetivos de la Directiva Marco del Agua de la Unión Europea. Por ello, resulta imprescindible invertir en la reposición y mejora de las infraestructuras. Las tarifas deberían cubrir la totalidad de los costes del servicio, desde la creación de la infraestructura hasta su conservación y reposición, teniendo en cuenta unas necesidades anuales de inversión de 4.000 millones de euros anuales durante los próximos diez años (Asociación de Ingenieros de Caminos, Canales y Puertos y de la Ingeniería Civil, 2019).

Por lo tanto, se concluye que el grado de cumplimiento del principio de recuperación de costes acuñado en la Directiva Marco del Agua es desigual de unos territorios a otros $y$, sin duda, esto genera una desigual disponibilidad de recursos para acometer las inversiones necesarias para, en primer lugar, asegurar la reposición de las infraestructuras existentes del ciclo urbano del agua (Asociación Española de Empresas Gestoras de los Servicios de Agua Urbana, 2018) y, de manera más urgente, garantizar el cumplimiento de la Directiva sobre depuración de aguas residuales urbanas. De hecho, como ya se ha mencionado más arriba, España ya ha sido condenada, por el Tribunal de Justicia de la Unión Europea, a pagar, a partir del 25 de julio de 2018, 10,95 millones de euros por cada semestre que persistan los $\triangleright$ 
incumplimientos que considera acreditados, además de una multa coercitiva inicial de 12 millones de euros (Tribunal de Justicia de la Unión Europea, 2018), y se corre el riesgo de incurrir en sanciones adicionales. Probablemente, este desigual grado de cumplimiento se debe a las reticencias de ciertas autoridades locales y autonómicas a aplicar, por consideraciones relacionadas con la asequibilidad, el principio de recuperación de los costes de un recurso escaso -especialmente en nuestro país - y medioambientalmente valioso como es el agua. Como consecuencia de ello, los recursos son, con frecuencia, insuficientes para cubrir las necesidades de inversión y de operación.

\section{Conclusiones}

Los pagos por uso podrían contribuir a mejorar la sostenibilidad del modelo de financiación de algunos servicios públicos, especialmente aquellos vinculados a infraestructuras. Dado que existen restricciones a la hora de acudir a otras fuentes de financiación, como la deuda pública, sería necesario, para realizar un uso óptimo de los escasos recursos públicos y garantizar la calidad y el mantenimiento de los servicios públicos vinculados a infraestructuras, disponer de un modelo estable y de largo plazo para su provisión que se apoye en el pago por su uso de las mismas.

Sin embargo, en España, la manera de abordar esta cuestión en algunos sectores ha sido poco coherente. Hasta ahora se han adoptado soluciones distintas para cada sector, con un enfoque ad hoc y errático. Se echa en falta un análisis global con un enfoque de política económica, lo cual ha llevado a una ausencia de consistencia en la aproximación a esta cuestión. Todo esto lleva a un modelo desdibujado de provisión de servicios públicos que puede no ser ni sostenible ni justo.

En el sector del transporte, no parece viable avanzar en la descarbonización del sector en España sin la generalización del pago por uso de las carreteras. Los vehículos deberían pagar en función de los costes de conservación y de los costes externos que generan, imponiendo tarifas más altas a los vehículos pesados, aunque posiblemente con una senda de aproximación gradual hasta el nivel que les corresponda pagar. El pago por el uso de las carreteras debería ser una fuente recurrente de recursos que permitiría mejorar el estado de conservación de las vías ${ }^{10}$. Potencialmente, podría generar recursos adicionales para afrontar otros costes indirectos de las carreteras u otros costes del proceso de descarbonización del sector. La tarificación solo de las vías de alta capacidad, por otro lado, puede tener el inconveniente de incentivar el uso de carreteras alternativas - nacionales o secundarias- con mayores índices de siniestralidad. Esto se debería también tener en cuenta a la hora de fijar los precios por el uso de las infraestructuras y hace recomendable ampliar el despliegue progresivo del sistema de cobro a otras vías, siempre que sea socialmente eficiente hacerlo.

En el caso del medioambiente, especialmente del agua, las políticas de precios actuales no aseguran la recuperación de los costes de los servicios relacionados con el agua, como exige la Directiva Marco del Agua. A pesar de ello y de las sanciones ya impuestas $\square$

\footnotetext{
10 Y para permitir la recuperación del coste de las propias inversiones necesarias para desplegar el sistema de cobro por uso de las carreteras, que podría pasar por sistemas de arcos que permitan la lectura de dispositivos que los vehículos llevarían a bordo, sin hacer necesario que el vehículo se detenga, como ocurre con las instalaciones tradicionales de
} peaje. 
por el Tribunal de Justicia de la Unión Europea, los Gobiernos autonómicos y locales son reacios a incrementar la recuperación de costes con cargo a los usuarios. Como consecuencia de ello, los recursos son, con frecuencia, insuficientes para cubrir las necesidades de inversión y de operación. Por lo tanto, se están retrasando inversiones urgentes en el sector. De hecho, se está constatando un envejecimiento de las infraestructuras.

Como se ha indicado, los cargos por uso son sensibles políticamente por preocupaciones relacionadas con la equidad y asequibilidad. Sin embargo, estas deberían abordarse por medio de políticas asistenciales reforzadas, diseñadas de manera que se dirijan a las personas que realmente las necesiten, en lugar de mediante la opción de no cobrar a nadie por el uso de las infraestructuras. Los cargos por uso permiten que la financiación de las infraestructuras no tenga que recaer sobre el conjunto de los contribuyentes, incluidos los de menores rentas y riqueza que, probablemente, hacen un menor uso de las mismas, así como señalizar mejor las externalidades que producen.

En resumen, la satisfacción de las necesidades de la población por medio de servicios públicos de calidad requiere un grado importante de inversiones y mantenimiento. Frente a otras fuentes de financiación, como la deuda pública, un modelo prudente y estable para la provisión de servicios públicos, con un enfoque de largo plazo y basado en pagos por parte de los usuarios, contribuiría a realizar un uso óptimo de los recursos públicos escasos y garantizar la provisión de servicios de calidad y su sostenibilidad. Un modelo así debería venir acompañado de políticas asistenciales reforzadas y dirigidas con mayor precisión a quienes realmente las necesitan.

\section{Bibliografía}

ADIF (2019). Cuentas auditadas a 31 de diciembre de 2018, página 98. http://www.adif.es/es_ES/ compromisos/doc/CCAA-ADIF-2018-inf-auditoria-gestion.pdf

ADIF Alta Velocidad (2019). Cuentas auditadas a 31 de diciembre de 2018, página 94. http://www. adifaltavelocidad.es/es_ES/compromisos/doc/ CCAA2018.pdf

AENA (2019). Cuentas anuales consolidadas a 31 de diciembre de 2018, página 67. http://www.aena.es/ csee/ccurl/757/276/Informe-auditoria-cuentasanuales-consolidadas-informe-gestion-consolidado-2018-Aena-sociedades-dependientes.pdf

Asociación de Ingenieros de Caminos, Canales y Puertos y de la Ingeniería Civil (2019). Las obras y servicios públicos a examen. España, informe 2019. http://ingenieria-civil.org/informe-2019/

Asociación Española de Empresas Gestoras de los Servicios de Agua Urbana (AGA) y Asociación Española de Abastecimiento de Agua y Saneamiento (AEAS) (2018). Datos sobre los servicios del agua urbana en España. Resultados del XV Estudio Nacional de Suministro de Agua Potable y Saneamiento en España 2018. Nota de Prensa, páginas 4 y 5 . https://www.asoaeas. $\mathrm{com} /$ ?q=content/nota-de-prensa-del-xv-estudionacional

AT Kearney (septiembre de 2018). Hacia un modelo social y sostenible de infraestructuras viarias en España. https://seopan.es/wp-content/uploads/ 2016/09/AT-KEARNEY_Hacia-un-modelo-socialy-sostenible-de-infraestructuras-viarias-en-Espa\%C3\%B1a.pdf

Comisión Europea (28 de marzo de 2011). Libro Blanco. Hoja de ruta hacia un espacio único europeo de transporte: por una política de transportes competitiva y sostenible. https://eur-lex. europa.eu/legal-content/ES/TXT/PDF/?uri= CELEX:52011DC0144\&from=EN

Comisión Europea (14 de diciembre de 2017). Commission staff working document accompanying $\triangleright$ 
the document report from the Commission to the European Parliament, the Council, the European Economic and Social Committee and the Committee of the Regions. Ninth Report on the implementation status and the programmes for implementation (as required by Article 17) of Council Directive 91/271/EEC concerning urban waste water treatment. https://eur-lex.europa.eu/ legal-content/EN/TXT/?qid=1560264152610\& uri=CELEX:52017SC0445

Comisión Europea (26 de febrero de 2019). Commission Staff Working Document. Second River Basin Management, Plans - Member State: Spain. https://eur-lex.europa.eu/legal-content/EN/TXT/ PDF/?uri=CELEX:52019SC0042\&from=en

Comisión Europea (26 de febrero de 2019). Report from the Commission to the European Parliament and the Council on the Implementation of the Water Framework Directive (2000/60/EC) and the Floods Directive (2007/60/EC). Second River Basin Management Plans First Flood Risk Management Plans. https://eur-lex.europa.eu/legal-content/EN/TXT/?qid=1560243396132\& uri=CELEX:52019DC0095

Comisión Europea (11 de diciembre de 2019). Communication from the Commission to the European Parliament, the European Council, the Council, the European Economic and Social Committee and the Committee of the Regions. The European Green Deal. https://eur-lex.europa.eu/resource.html?uri=cellar:b828d165-1c2211ea-8c1f-01aa75ed71a1.0002.02/DOC_1\& format=PDF

Comisión Nacional de los Mercados y de la Competencia (11 de diciembre de 2019). Resolución de supervisión de las tarifas aeroportuarias aplicables por AENA, S.M.E., S.A. en el ejercicio 2020, páginas 20 y 21 . https://www.cnmc.es/sites/default/files/2775990.pdf

Delegación del Gobierno en las Sociedades Concesionarias de Autopistas Nacionales de Peaje (2018). Informe 2017 sobre el sector de autopistas de peaje en España, páginas 7 y 129. España: Ministerio de Fomento. https://apps.fomento.gob.es/ CVP/handlers/pdfhandler.ashx?idpub=ICW039
De Rus, G., Campos, J., y Nombela, G. (2003). Economía del Transporte. Antoni Bosch, editor.

Directiva 2000/60/CE del Parlamento Europeo y del Consejo de 23 de octubre de 2000 , por la que se establece un marco comunitario de actuación en el ámbito de la política de aguas.

Directiva 2011/76/UE del Parlamento Europeo y del Consejo, de 27 de septiembre de 2011, por la que se modifica la Directiva 1999/62/CE, relativa a la aplicación de gravámenes a los vehículos pesados de transporte de mercancías por la utilización de determinadas infraestructuras. Texto pertinente a efectos del EEE. https://eur-lex.europa.eu/legal-content/ES/TXT/PDF/?uri=CELEX: 32011 L0076\&from $=E S$

Ley 38/2015, de 29 de septiembre, del sector ferroviario, artículo 97. Boletín Oficial del Estado, núm. 234, de 30 de septiembre de 2015, páginas 88533 a 88634 . https://www.boe.es/boe/ dias/2015/09/30/pdfs/BOE-A-2015-10440.pdf

Ley 6/2018, de 3 de julio, de Presupuestos Generales del Estado para el año 2018, artículo 97. Boletín Oficial del Estado, núm, 161, de 4 de julio de 2018, páginas 66621 a 67354 . https://www. boe.es/boe/dias/2018/07/04/pdfs/BOE-A-20189268.pdf

Nicholson, W. (1997). Teoría Microeconómica. Principios básicos y aplicaciones. McGraw-Hill/Interamericana de España, SAU.

Observatorio del Transporte y la Logística en España (marzo de 2019). Informe Anual de 2018. Ministerio de Fomento. https://observatoriotransporte.fomento.es/recursos_otle/informe_otle_ 2018.pdf

Puertos del Estado (2019). Informe de Gestión de Sistema Portuario de Titularidad Estatal 2018. Ministerio de Fomento. http://www.puertos.es/ es-es/Documents/Informe\%20de\%20Gestion\% 202018.pdf

Real Decreto Legislativo 1/2001, de 20 de julio, por el que se aprueba el texto refundido de la Ley de Aguas. Boletín Oficial del Estado, núm. 176, $\triangleright$ 
de 24 de julio de 2001, páginas 26791 a 26817. https://www.boe.es/boe/dias/2001/07/24/pdfs/ A26791-26817.pdf

Tribunal de Justicia de la Unión Europea (2018). Sentencia del Tribunal de Justicia (Sala Octava) de 25 de julio de 2018. Asunto C-205/17.
Luxemburgo. http://curia.europa.eu/juris/celex. jsf?celex=62017CJ0205\&lang $1=$ en \&type $=T X$ T\&ancre $=$

Rodrik, D. (2015). Economics Rules. The Rights and Wrongs of the Dismal Science. WW Norton \& Company. 